\title{
Prevalence of Depression in Gynecologic and Breast Cancer Patients
}

\author{
Heather Dorich, PharmD Candidate Class of 2019ª , Jayne Pawasauskas PharmD, \\ BCPS $^{b}$, Britny Rogala PharmD, BCOP ${ }^{c}$, Kate Lally MD $^{d}$
}

a The University of Rhode Island College of Pharmacy, 7 Greenhouse Rd Kingston, RI 02881 USA (hdorich13@gmail.com)

${ }^{b}$ Department of Pharmacy Practice, University of Rhode Island College of Pharmacy, 7 Greenhouse Rd Kingston, RI 02881 USA

c Department of Pharmacy Practice, University of Rhode Island College of Pharmacy, 7 Greenhouse Rd Kingston, RI 02881 USA; Women and Infants Hospital 101 Dudley St, Providence, RI 02905 USA

d Harvard Medical School, Department of Psychosocial Oncology and Palliative Care, Dana Farber Cancer Institute, 450 Brookline Ave D-2016A Boston, MA 02215 USA

DOI: $10.47611 /$ jsr.v9i1.650

\section{Introduction}

Patients with advanced cancer often face a high symptom burden including both physical and emotional symptoms. [1] Cancer patients with more advanced disease or high symptom burdens can derive the most benefit from palliative care. The role of palliative care in these patients is to manage their symptoms and assist with discussions around goals of care. [2] In this population, many studies indicate that depression can be fairly common. [3-5] A systematic review of major depression in patients receiving palliative care indicated a prevalence of approximately $15 \%$. [3] Other studies, using meta-analysis, have shown ranges of depression anywhere from $5.1 \%$ to $24 \%$. [4,5] Due to the high likelihood of multiple comorbidities and stressful life events in patients with advanced cancer, it is possible that this subset of patients may be at higher risk for depression than the general population. [3] Data from the National Institute of Mental Health indicates that $6.7 \%$ of the US population had one major depressive episode in 2016. [6] In a population of patients with cancer, risk factors for and comorbidities associated with depression are lacking. This is of particular interest for palliative care patients, as the symptoms of advanced cancer such as fatigue, anorexia and weight loss can often resemble the symptoms of depression. [7] A greater understanding of comorbidities associated with depression is important in that it may lead to enhanced screening for depression and as a result, earlier treatment.

Studies have indicated that psychological distress can be common in patients with gynecologic cancers. [8] Depression in patients with gynecologic malignancies can be as high as 33-52\%. [9] We sought to explore the prevalence of depression in a unique population and to consider patient-specific variables associated with its diagnosis. Identification of specific patient characteristics associated with a depression diagnosis could be used in the future to 
indicate patients with the greatest need for screening. Enhanced screening for depression could, in turn, lead to improved quality of life via earlier diagnosis and treatment.

\section{Materials and Methods}

The Women and Infants Institutional Review Board reviewed and approved this study. The primary objective was to quantify the prevalence of depression among palliative care patients at a women's oncology hospital. The secondary objective was to identify specific patient comorbid factors that are associated with the diagnosis of depression in the cohort.

This retrospective cohort study evaluated patients admitted to Women and Infants Hospital in Providence, Rhode Island, between 9/30/16 and 9/30/17. All data was collected through retrospective review of electronic medical records. Records were included if the patient was at least 18 years of age and had received a formal consultation request for the palliative care service. Patients were required to have had an inpatient stay in conjunction with the consultation. Records were excluded if vital study-related data was omitted or unobtainable through electronic records, or if the patient never received an actual consult by the palliative care service. If the patient was hospitalized multiple times over the course of the study, the most recent hospitalization was used for analysis.

Data analyzed included demographics (age, gender, race, marital status, and religion), past medical history (documented diagnoses), body mass index, medication list, clinical progress notes, and specialist consultation notes. A diagnosis of depression was determined by an ICD code for depression, a clearly stated diagnosis of depression in a medical note, or administration of any drug therapy that is a clinically accepted treatment for depression. Types of cancer were specified for analysis, and differentiated between gynecological and non-gynecological. Presence of metastatic disease was documented. Anxiety was assessed as a unique condition, and other psychiatric conditions were identified and grouped. Each patient's Charlson Comorbidity Index score was calculated based on data collected. The Charlson Comorbidity Index is used to provide a weighted score of a person's burden of disease. Specific conditions are assigned scores (Table 1) which are then added together based on a patient's comorbidities.

Table 1: Charlson Comorbidity Index

\begin{tabular}{|c|c|}
\hline Assigned weights for diseases & Conditions \\
\hline 1 & Myocardial infarct \\
& Congestive heart failure \\
& Peripheral vascular disease \\
& Cerebrovascular disease \\
& Dementia \\
& Chronic pulmonary disease \\
& Connective tissue disease \\
& Ulcer disease \\
& Mild liver disease \\
\hline
\end{tabular}




\begin{tabular}{|c|c|}
\hline 2 & $\begin{array}{c}\text { Hemiplegia } \\
\text { Moderate or severe renal disease } \\
\text { Diabetes with end organ damage } \\
\text { Any tumor } \\
\text { Leukemia } \\
\end{array}$ \\
\hline 3 & Lymphoma \\
\hline 6 & Moderate or severe liver disease \\
\hline & Metastatic solid tumor \\
\hline
\end{tabular}

Statistical analyses were performed using SPSS (IBM corp, version 23). Descriptive statistics were used to report frequencies and averages of demographic variables. Comorbidities were grouped by organ system and assigned a nominal categorization score for the purpose of analyses. Diagnoses of depression were analyzed as dichotomous variables and Chi-square tests were used to evaluate relationships between depression and the presence of other individual comorbidities. Fisher's Exact tests were used for variables with counts fewer than 5. Similarly, variables of race, marital status and religion were categorized as dichotomous variables. Mann Whitney U was used to analyze differences between cohorts for continuous variables, including age, body mass index, number of medications utilized, and number of consultations to specialized health care providers.

\section{Results}

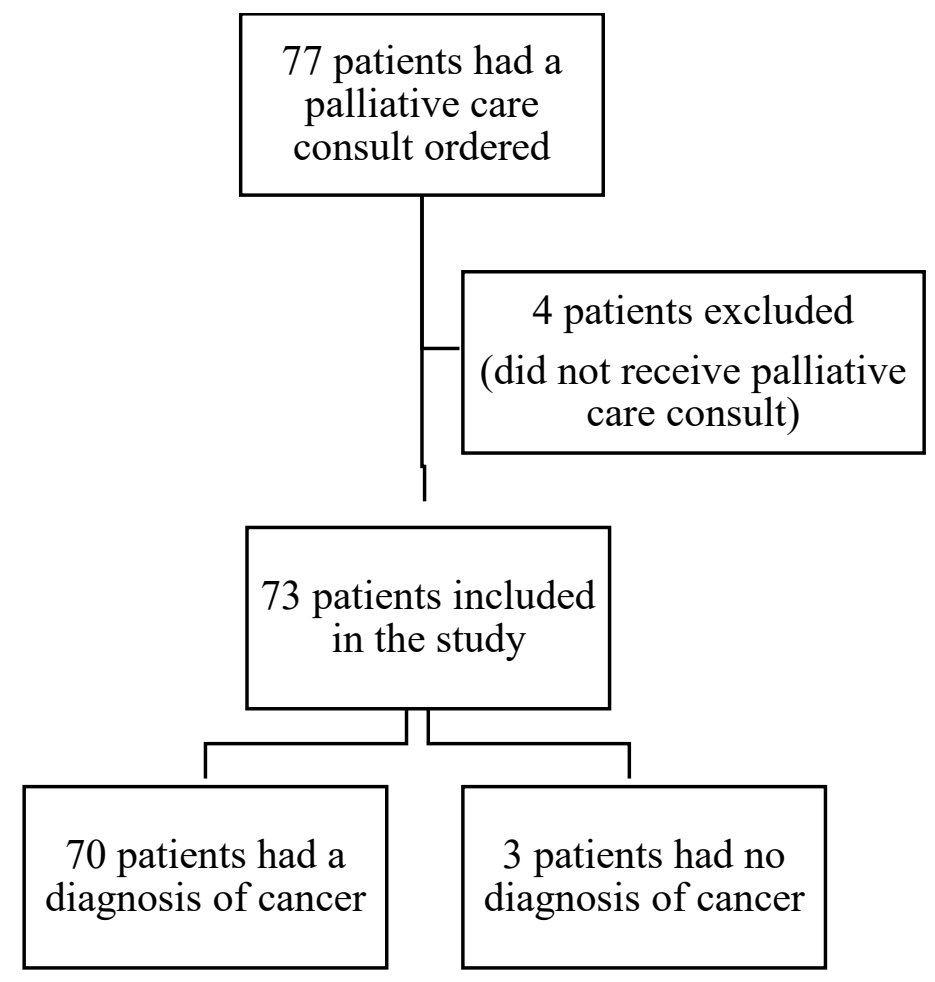

Figure 1: Study Profile 
A total of 77 patients were screened and 73 met inclusion criteria (Figure 1). Of these, 54.8\% had a diagnosis of depression (Table 2). Due to the nature of the population, all patients in the sample were female. The average age of the sample was 61.5 years (+/- 13.3) and the majority were Caucasian $(82.2 \%)$. Married patients comprised the largest reported group regarding marital status (49.3\%), and Catholic religion was the most commonly reported $(45.2 \%)$ religious denomination. All but 3 patients had a documented diagnosis of cancer. The remaining 3 were being evaluated and receiving diagnostic tests to determine cancer diagnoses during the study period. The most prevalent type of cancer was ovarian (26\%), and $45.2 \%$ of the total sample had metastatic disease. A slight majority of the sample had a diagnosis of anxiety or other psychiatric condition (56.1\%). On average, patients used $13.4(+/-$ 5.5) medications, and received $7(+/-4.8)$ consults from any service.

Table 2: Results

\begin{tabular}{|c|c|c|c|c|}
\hline Variable & $\begin{array}{l}\text { Total Sample } \\
\qquad(\mathrm{n}=73)\end{array}$ & $\begin{array}{c}\text { Non-depressed co- } \\
\text { hort } \\
(n=33)\end{array}$ & $\begin{array}{l}\text { Depressed cohort } \\
\qquad(n=40)\end{array}$ & p-value \\
\hline Age (average, in years \pm SD) & $61.5 \pm 13.3$ & $64.6 \pm 13.8$ & $58.9 \pm 12.5$ & 0.062 \\
\hline Body mass index $( \pm \mathrm{SD})$ & $28.2 \pm 9$ & $28.6 \pm 7.3$ & $27.9 \pm 10.4$ & 0.320 \\
\hline Charlson Comorbidity Score & $4.95 \pm 2.65$ & $5.33 \pm 2.57$ & $4.63 \pm 2.70$ & 0.258 \\
\hline Metastatic disease* & 33 & 19 & 14 & 0.045 \\
\hline Breast cancer* & 7 & 3 & 4 & 0.608 \\
\hline Endometrial cancer* & 17 & 11 & 6 & 0.059 \\
\hline Cervical cancer* & 10 & 3 & 7 & 0.245 \\
\hline Ovarian cancer* & 19 & 10 & 9 & 0.312 \\
\hline Fallopian cancer* & 5 & 0 & 5 & 0.044 \\
\hline Peritoneal cancer* & 5 & 3 & 2 & 0.408 \\
\hline Other cancer type* & 9 & 4 & 5 & 0.623 \\
\hline Other psychiatric condition* & 41 & 10 & 31 & $<0.0001$ \\
\hline Diabetes* & 16 & 7 & 9 & 0.562 \\
\hline Disabling disease* & 33 & 12 & 21 & 0.127 \\
\hline Arthritis* & 21 & 9 & 12 & 0.503 \\
\hline Substance Use Disorder* & 3 & 2 & 1 & 0.427 \\
\hline $\begin{array}{l}\text { Number of Medications } \\
\text { (average } \pm \text { SD) }\end{array}$ & $13.4 \pm 5.5$ & $12.9 \pm 5.6$ & $13.9 \pm 5.5$ & 0.530 \\
\hline $\begin{array}{c}\text { Number of Consults (average } \\
\pm \mathrm{SD})\end{array}$ & $7 \pm 4.8$ & $8.2 \pm 5.1$ & $6.0 \pm 4.3$ & 0.034 \\
\hline Race & & & & \\
\hline Caucasian & 60 & 25 & 35 & 0.159 \\
\hline African American & 6 & 3 & 3 & 0.566 \\
\hline Hispanic & 1 & 1 & 0 & 0.452 \\
\hline
\end{tabular}




\begin{tabular}{|c|c|c|c|c|}
\hline Asian & 2 & 2 & 0 & 0.201 \\
Alaskan Native & 1 & 1 & 0 & 0.452 \\
Other & 3 & 1 & 1 & 0.703 \\
\hline Marital status & 15 & 7 & 8 & 0.562 \\
Single & 36 & 14 & 22 & 0.202 \\
Married & 7 & 4 & 3 & 0.392 \\
Divorced & 13 & 8 & 5 & 0.159 \\
Widowed & 2 & 0 & 2 & 0.297 \\
Separated & 33 & 16 & 17 & \\
Religion & 16 & 7 & 9 & 0.391 \\
Catholic & 1 & 0 & 1 & 0.562 \\
Non-Catholic Christian & 1 & 1 & 0 & 0.548 \\
Jewish & 1 & 0 & 1 & 0.452 \\
Mormon & 11 & 6 & 5 & 0.548 \\
Orthodox & 10 & 3 & 7 & 0.363 \\
Unknown & & & & 0.245 \\
None & & 0 & & \\
\hline
\end{tabular}

* results expressed as number of patients with specified variable per cohort

In our analysis of variables between the depressed and non-depressed cohorts, we found significant differences in rates of depression for patients with metastatic disease (35\% vs. 57.6\%, p=0.045), cancer of the fallopian tube (12.5\% vs. $0, \mathrm{p}=0.044)$, and those with anxiety or other psychiatric condition $(77.5 \%$ vs. $25 \%, \mathrm{p}<0.0001)$. Patients who did not have a diagnosis of depression received more consults than those without depression (8.2 +/- 5.1 vs 6.0 +/- 4.3, $\mathrm{p}=0.034)$. Patients with depression tended to be younger, although the difference did not reach statistical significance. (58.9 vs. 64.6 years, $\mathrm{p}=0.062$ ). There were no significant differences between the depressed and nondepressed cohorts with regard to body mass index, race, marital status, religion, Charlson Comorbidity Score, remaining cancer types, substance use disorder, or the number of medications used.

A finding of statistical significance representing the difference in number of consults to specialized health care providers between the depressed versus non-depressed cohorts prompted an ad hoc analysis. Regression analyses were applied to explore possible relationships between number of consults received and other study variables collected. There were no significant associations identified between number of consults received and Charlson Comorbidity scores, or the presence of metastatic disease, anxiety, psychiatric conditions, or disabling disease.

\section{Discussion}

The results of this study indicate a high prevalence of depression among women with gynecologic and breast cancers. Associated conditions could potentially be used as predictive factors to indicate an evaluation for depression may be needed. Moreover, our study indicates that screening for depression may not occur as frequently as necessary. Out of the 73 patients included in this study, 54.8\% were found to have a diagnosis of depression. Depression 
itself was found to be independently associated with the presence of anxiety, cancer of the fallopian tube, and nonmetastatic disease. Anxiety and depression have long been known to be highly comorbid in both the cancer and noncancer populations. [10] Although, the association between depression and metastatic disease may seem common sense since patients with metastatic disease often have worse disease, the quantifiable data from our study indicates that this is not the case. The Charlson Comorbidity score was not found to be positively associated with the diagnosis of depression.

Our sample's prevalence of depression at $54.8 \%$ is markedly greater than the $6.7 \%$ estimated prevalence in the general US population. [6] The prevalence of depression in our sample is similar to that of another small, singleinstitution study, which found a $61 \%$ prevalence among patients with metastatic breast cancer. [11] A systematic review of women with metastatic breast cancer cited a rate of depression of up to $32 \%$ [3] and a systematic review of palliative care patients that indicated a $15 \%$ median prevalence of depression. [12] Depression can negatively affect a cancer patient's quality of life and functional status. [6,13] Cancer patients with depression have a lower health-related quality of life (HRQL) than their non-depressed counterparts while those with depression and anxiety have worse HRQL than patients who have depression with no anxiety. [10] A study using the Behavioral Risk Factor Surveillance System surveys found that people with depression had a life expectancy that was 16.4 years fewer at age 18 than people who were not depressed. The quality adjusted life years at age 18 was also 28.9 years fewer in people with depression. [14] The large variations in prevalence of depression within the palliative care and gynecologic and breast cancer population could indicate there is a substantial portion of patients that are dealing with undiagnosed depression, and as such, could be suffering from the effects of depression on their quality of life and functional status.

A significant financial burden is associated with depression for both the patient and society as a whole. The financial burden placed on patients and society includes direct medical costs, suicide-related mortality, and indirect workplace costs due to absenteeism and presenteeism. [15] The direct medical costs attributed to individuals with major depressive disorder in 2010 was $\$ 98.8$ billion with an additional $\$ 9.6$ billion in suicide-related costs, and $\$ 102$ billion in indirect workplace costs. [15] Patient quality of life and the financial burden associated with depression are two reasons that diagnosis and treatment are vital, and perhaps more so for the cancer population. The impact of depression on patients' quality of life is important because psychiatric morbidity may often go unrecognized and/or misclassified in cancer patients, leading to lower rates of treatment. [16]

Historically, depression has been an underdiagnosed and undertreated condition in the cancer patient population. [10] This may in part be due to the high physical and emotional symptom burden often seen in patients with advanced cancer, some of which are similar to symptoms of depression. [1] Another potential explanation for missed diagnoses is a lack of provider education on depression screening in complex cancer patients and limited palliative care resources. [17]

The criteria required to meet a diagnosis of depression may also differ based on the provider and the situation. Due to the retrospective nature of our study, information analyzed was previously documented in the patients' charts. As such, background information specifying the timing of a diagnosis of depression or the associated symptoms, was not available. Furthermore, there is no standard method to classify patients in terms of their trajectory of palliative care services. For example, a patient may be newly diagnosed with an incurable condition and just beginning to utilize palliative care services, whereas another patient may be nearing hospice care. Moreover, the timing of 
the cancer diagnosis and metastatic disease were not known in relation to each other, nor correlated with the depression diagnosis. Therefore, a patient who was hospitalized and received a new diagnosis of metastatic disease would be documented as such for the purpose of analysis, however, the development and proper diagnosis of depression could occur weeks to months later. The timing of depression onset compared to cancer diagnosis could explain why patients with metastatic disease were found to be less likely to have depression in our study.

Although we tend to assume that patients with worse prognoses will be more prone to depression, findings of this study indicate that simply considering their physical status may not be the most effective way to determine if a patient with gynecological or breast cancer is likely to become depressed. The Charlson Comorbidity score, which helps to stratify the degree of comorbid illness burden, was not found to be associated with an increased incidence of depression. The findings of this study indicated that those with a higher score, indicating a greater burden of comorbid illness, may be less likely to have depression than those with a lower score, although the findings were not statistically significant.

The associations found in this study could be used to inform health care practitioners of indicators to initiate screening patients for depression or depression itself. For example, the presence of cancer of the fallopian tube could prompt for a depression screening. Pharmacists could also screen medication lists to determine if a diagnosis of depression may have been missed or a patient needs to be screened for depression. The presence of psychiatric or anxiety medications could be indicators for this to occur. Although distress screening is recommended for all cancer patients, it is not routinely performed in clinical practice. [18] This study further highlights the need for routine distress and depression screening, given the high prevalence of depression among gynecologic and breast cancer patients in this study.

The data from this study is limited by the small sample size and retrospective nature of the data. Our study occurred in a single institution over a one-year period of time. The hospital is specific to women and infant care, and as such the population of the study was entirely female with breast or gynecologic related malignancies. The retrospective nature of the study required the researchers to rely on records as they existed at the time of the study. Limitations of this study also include the requirement of a palliative care consult for inclusion in the analysis. Not all patients on the oncology service receive palliative care consults as these are often triggered by a need for a goals of care discussion or symptom management.

With more research into the association between depression and patients with gynecological or breast cancer, patient-specific factors could be discovered that are predictive of depression. This information could then be operationalized to optimize the tools that currently help practitioners screen oncology patients for depression. With increased screening and detection of depression, its diagnosis in cancer patients could be made more quickly, with a resulting opportunity to increase the quality of life in these patients.

\section{References}

[1] Friedlander, M. L., Stockler, M., O'Connell, R., Voysey, M., Oza, A., Gillies, K...Symptom Benefit Study Group (2014). Symptom burden and outcomes of patients with platinum resistant/refractory recurrent ovarian cancer: a reality check: results of stage 1 of the gynecologic cancer intergroup symptom benefit study. International journal of gynecological cancer, 24(5), 857-64. doi: 10.1097/IGC.0000000000000147. 
[2] World Health Organization (2018). Palliative Care. Retrieved from: http://www.who.int/en/news-room/factsheets/detail/palliative-care

[3] Hotopf, M., Chidgey, J., Addington-Hall, J., \& Ly, K.L. (2002). Depression in advanced disease: a systematic review Part 1. Prevalence and case finding. Palliative Medicine, 16(2), 81-97.

[4] Mitchell, A.J., Chan, M., Bhatti, H., Halton, M., Grassi, L., Johansen, C., \& Meader, N. (2012). Prevalence of depression, anxiety, and adjustment disorder in oncological, haematological, and palliative-care settings: a metaanalysis of 94 interview-based studies. The Lancet. Oncology, 12(2), 160-74. doi: 10.1016/S1470-2045(11)70002-X.

[5] Krebber, A.M., Buffart, L.M., Kleijn, G., Riepma, I.C., de Bree, R., Leemans, C.R... Verdonck-de Leeuw, I.M. (2014). Prevalence of depression in cancer patients: a meta-analysis of diagnostic interviews and self-report instruments. Psycho-oncology, 23(2),121-30. doi: 10.1002/pon.3409.

[6] National Institute of Mental Health (2018). Major Depression. Retrieved from: https://www.nimh.nih.gov/health/statistics/major-depression.shtml.

[7] Newport, D.J., \& Nemeroff, C.B. (1998). Assessment and treatment of depression in the cancer patient. Journal of Psychosomatic Research, 45(3),215-37.

[8] Cassedy, H.F., Tucker, C., Hynan, L.S., Phillips, R., Adams, C., Zimmerman, M.R.... Stringer, C.A. (2018). Frequency of psychological distress in gynecologic cancer patients seen in a large urban medical center. Proceedings (Baylor University. Medical Center),31,161-164. doi: 10.1080/08998280.2018.1440857.

[9] Klügel, S., Lücke, C., Meta, A., Schild-Suhren, M., Malik, E., Philipsen, A., \& Muller, H. H. (2017). Concomitant psychiatric symptoms and impaired quality of life in women with cervical cancer: a critical review. International Journal of Women's Health, 9,795-805. doi: 10.2147/IJWH.S143368.

[10] Brown, L.F., Kroenke, K., Theobald, D.E., Wu, J., \& Tu, W. (2010). The association of depression and anxiety with health-related quality of life in cancer patients with depression and/or pain. Psycho-oncology, 19(7),734-41. doi: $10.1002 /$ pon. 1627.

[11] Alacacioglu, A., Yavuzsen, T., Dirioz, M., \& Yilmaz, U. (2009). Quality of life, anxiety and depression in Turkish breast cancer patients and in their husbands. Medical Oncology, 26(4),415-9. doi: 10.1007/s12032-0089138-z.

[12] Caplette-Gingras, A., \& Savard, J. (2008). Depression in women with metastatic breast cancer: a review of the literature. Palliative \& Supportive Care,6(4),377-87. doi: 10.1017/S1478951508000606. 
[13] Kroenke, K., Theobald, D., Wu, J., Loza, J.K., Carpenter, J.S., \& Tu, W. (2010). The association of depression and pain with health-related quality of life, disability, and health care use in cancer patients. Journal of Pain and Symptom Management, 40(3),327-41. doi: 10.1016/j.jpainsymman.2009.12.023.

[14] Jia, H., Zack, M.M., Thompson, W.W., Crosby, A.E., \& Gottesman, I.I. (2015). Impact of depression on quality-adjusted life expectancy (QALE) directly as well as indirectly through suicide. Social Psychiatry and Psychiatric Epidemiology, 50, 939-49. doi:10.1007/s00127-015-1019-0.

[15] Greenberg, P.E., Fournier, A.A., Sisitsky, T., Pike, C.T., \& Kessler, R.C. (2015). The economic burden of adults with major depressive disorder in the United States (2005 and 2010). The Journal of Clinical Psychiatry, 76(2), 155-62. doi: 10.4088/JCP.14m09298.

[16] Fallowfield, L., Ratcliffe, D., Jenkins, V., \& Saul J. (2001). Psychiatric morbidity and its recognition by doctors in patients with cancer. British Journal of Cancer, 84(8), 1011-5.

[17] Thomas, R.A., Curley, B., Wen, S., Zhang, J., Abraham, J., \& Moss, A.H. (2015). Palliative Care Training during Fellowship: A National Survey of U.S. Hematology and Oncology Fellows. Journal of Palliative Medicine, 18(9), 747-51. doi: 10.1089/jpm.2015.0035.

[18] Holland, J.C., Deshields, T.L., Andersen, B., Braun, I., Breitbart, W.S., Brewer, B.W., et al. Distress Management. National Comprehensive Cancer Network Clinical Practice Guidelines in Oncology. Version 2.2018. 